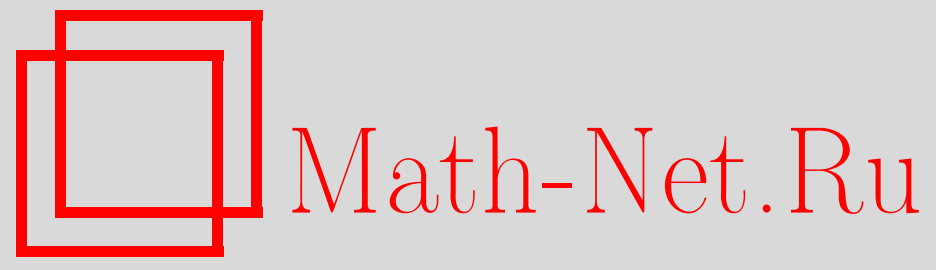

Ю. В. Гришко, Об асимметрично разложимых группах, $M a$ тем. заметки, 2003, том 74, выпуск 3, 475-477

DOI: https://doi.org/10.4213/mzm595

Использование Общероссийского математического портала Math-Net.Ru подразумевает, что вы прочитали и согласны с пользовательским соглашением http://www.mathnet.ru/rus/agreement

Параметры загрузки:

IP : 18.207 .199 .55

26 апреля 2023 г., 17:59:16

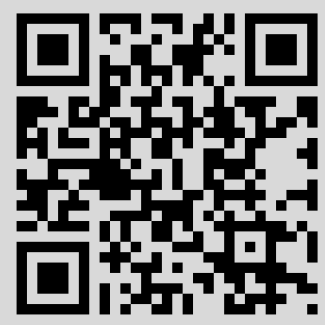




\section{ОБ АСИММЕТРИЧНО РАЗЛОЖИМЫХ ГРУППАХ}

\section{Ю. В. Гришко}

Подмножество $A$ группы $G$ назьвается симметричным относительно элемента $g \in G$, если $A=g A^{-1} g$. Группа назьвается асимметрично разложимой, если ее можно разбить на два подмножества, не содержащие бесконечных симметричных подмножеств. Абелева группа асимметрично разложима тогда и только тогда, когда она почти цик лическая либо счетная периодическая с конечным числом элементов порядка 2 [1]. Группа называется почти чиклической, если она содержит циклическую подгруппу конечного индекса.

В неабелевом случае исследование группы на предмет асимметричной разложимости значительно сложнее. Так, было неизвестно, являются ли асимметрично разложимыми свободная группа с двумя образующими, а также любая бесконечная конечно порожденная периодическая группа. О второй группе было неизвестно даже, найдется ли сколь угодно большое конечное одноцветное симметричное подмножество для произвольного ее 2-раскрашивания. Это частный случай $(r=2)$ следующих двух вопросов.

(1) Верно ли, что для любого $r$-раскрашивания свободной группы с двумя образующими найдется бесконечное одноцветное симметричное подмножество (Р. И. Григорчук, [2, problem 1.2])?

(2) Верно ли, что для любого $r$-раскрашивания произвольной бесконечной группы найдется сколь угодно большое конечное одноцветное симметричное подмножество (И. В. Протасов, [2, problem 1.6])?

Второй вопрос редуцируется к бесконечным конечно порожденным группам конечного периода следующим образом. Если порядки элементов группы неограничены в совокупности, то применяем теорему ван дер Вардена: на множестве натуральных чисел существует функция $n(r, l)$ такая, что для любого $r$-раскрашивания множества первых $n(r, l)$ натуральных чисел найдется одноцветная арифметическая прогрессия длины $l$. Если группа имеет конечный период и локально конечна, то выделяем в ней бесконечную абелеву подгруппу (теорема Каргаполова-Холла-Кулатилаки) и применяем теорему Грэхема-Либа-Росчайльда: для любого $r$-раскрашивания бесконечной абелевой группы конечного периода найдется сколь угодно большой одноцветный смежньй класс по конечной подгруппе. Если же группа имеет конечный период и отлична от локально конечной, то она содержит бесконечную конечно порожденную группу конечного периода.

Мы докажем, что если коммутант группы содержит конечно порожденную подгруппу, отличную от почти циклической, то она не является асимметрично разложимой. Из этого следует, что асимметрично разложимыми не являются свободная группа с двумя образующими и бесконечная конечно порожденная периодическая группа.

В нашем доказательстве важную роль играет тот нетривиальный факт, что почти циклической является любая группа линейного роста. Действительно, каждая группа полиномиального роста содержит нильпотентную подгруппу $G$ конечного индекса [2] и степень $d$ полинома вычисляется при помощи нижнего центрального ряда

$$
G=G_{1}>G_{2}>\cdots, \quad G_{k+1}=\left[G, G_{k}\right]
$$


по формуле

$$
d=\sum_{k \geqslant 1} k \cdot r_{0}\left(G_{k} / G_{k+1}\right)
$$

где $r_{0}(A)$ - ранг без кручения абелевой группы $A[3]$. Если $d=1$, то ранг первой секции ряда равен 1 , а ранги остальных секций равны 0 . Так как $G$ конечно порождена и нильпотентна, то конечно порождены и все члены ряда, а значит, все секции - конечно порожденные абелевы группы. Таким образом, первая секция почти цик лическая, а остальные конечные, а следовательно, и вся группа $G$ почти циклическая.

TЕорема. Пусть әруппа $G$ содержит конечное подмножество $X=X^{-1} \ni 1$ maкое, что подгруппа $[X, X]=\langle[x, y]: x, y \in X\rangle$ отлична от почти ииклической. Тогда для любого 2-раскрашивания $G$ найдется бесконечное одночветное подмножество, симметричное относительно некоторого элемента из $X^{2}$.

ДоКАЗАТЕЛЬСтво. Допустим противное. Тогда найдется конечное $F_{0} \subset G$ такое, что для любых $g \in G \backslash F_{0}$ и $x \in X^{2}$ элементы $g$ и $z g^{-1} z$ разных цветов. Но тогда, поскольку цветов лишш два, найдется конечное $F \supseteq F_{0}$ такое, что для любых $g \in G \backslash F$ и $z \in X^{2}$ элементы $g$ и $z g z=\left(z^{-1} g^{-1} z^{-1}\right)^{-1}$ одного цвета. Переходы вида $g \rightarrow z g z$ будем называть элементарными. Отметим, что для любых конечного $K \subset G$ и натурального $s$ существует лишь конечное число элементов $G$, из которых можно добраться до $K$ за $\leqslant s$ элементарных переходов.

Идея доказательства такова. Мы выберем некоторый элемент $a \in H \backslash F$ и перейдем с помощью элементарных переходов от $a$ к $a^{-1}$, минуя $F$, что и будет противоречием. Для того, чтобы миновать $F$, мы выберем некоторый элемент $b \in H$ и перейдем вначале от $a$ к $a b$, затем от $a b$ к $b a^{-1}$, а затем от $b a^{-1}$ к $a^{-1}$. Более детально, обозначим

$$
U=\{[x, y]: x, y \in X\}, \quad H=\langle U\rangle=[X, X] .
$$

Пока еще не выбранный элемент $a$ запишем в виде $a=z_{1} \ldots z_{m}, z_{i} \in X$, а пока еще не выбранньй элемент $b$-в виде $b=u_{1} \ldots u_{n}, u_{j} \in U, u_{j}=\left[x_{j}, y_{j}\right], x_{j}, y_{j} \in X$. От $a$ к $a b$ перейдем следующим образом:

$$
\begin{aligned}
a & \rightarrow x_{1} a x_{1} \rightarrow y_{1} x_{1} a x_{1} y_{1} \rightarrow a x_{1} y_{1}\left(y_{1} x_{1}\right)^{-1} \\
& =a x_{1} y_{1} x_{1}^{-1} y_{1}^{-1}=a\left[x_{1}, y_{1}\right]=a u_{1} \rightarrow \cdots \rightarrow a u_{1} \ldots u_{n}=a b .
\end{aligned}
$$

Для того, чтобы перейти от $b a^{-1}$ к $a^{-1}$, вначале перейдем от $a b=z_{1} \ldots z_{m} b \mathrm{k} z_{m} \ldots z_{1} b$. Поясним лише, как перейти от $z_{1} \ldots z_{i-1} z_{i} z_{i+1} z_{i+2} \ldots z_{m} b$ к $z_{1} \ldots z_{i-1} z_{i+1} z_{i} z_{i+2} \ldots z_{m} b$ :

$$
\begin{aligned}
z_{1} \ldots z_{m} b & \rightarrow z_{2} \ldots z_{m} b z_{1}^{-1} \rightarrow \cdots \rightarrow z_{i+2} \ldots z_{m} b z_{1}^{-1} \ldots z_{i+1}^{-1} \\
& \rightarrow z_{i+1} z_{i} z_{i+2} \ldots z_{m} b z_{1}^{-1} \ldots z_{i-1}^{-1} \\
& \rightarrow z_{i-1} z_{i+1} z_{i} z_{i+2} \ldots z_{m} b z_{1}^{-1} z_{i-2}^{-1} \rightarrow \cdots \rightarrow z_{1} \ldots z_{i-1} z_{i+1} z_{i} z_{i+2} \ldots z_{m} b,
\end{aligned}
$$

а затем перейдем от $z_{m} \ldots z_{1} b$ к $b z_{m}^{-1} \ldots z_{1}^{-1}=b a^{-1}$. Как видим, в переходе от $a b$ к $b a^{-1}$ число элементарных переходов зависит лиш от $m$. И наконец, перейдем от $b a^{-1}$ к $a^{-1}$. Нам осталось выбрать элементы $a, b$.

Для каждого $g \in H$ через $l(g)$ обозначим наименьшую из длин разложений $g$ через элементы из $U$ :

$$
l(g)=\min \left\{n<\omega: g \in U^{n}\right\} .
$$

Отметим, что $l\left(g^{-1}\right)=l(g), l(g h) \leqslant l(g)+l(h)$, а значит, и $l(g h) \geqslant|l(g)-l(h)|$.

Выберем последовательость $\left\langle u_{n}\right\rangle_{n \in \mathbb{N}}$ в $U$ следующей леммой.

Лемма 1. Существует последовательность $\left\langle U_{n}\right\rangle_{n \in \mathbb{N}}$ в $U$ такая, ито $l\left(u_{1} \ldots u_{n}\right)=n$. 
ДоКАЗАТЕЛЬСтво. Для каждого неединичного $g \in H, l(g)=n$, зафикисируем минимальное разложение $g=u_{1}(g) \ldots u_{n}(g)$. Выберем последовательность $\left\langle g_{n}\right\rangle_{n \in \mathbb{N}}$ в $H$ такую, что $l\left(g_{n}\right) \geqslant n$ и $u_{1}\left(g_{n}\right)=u_{1}$ для некоторого $u_{1} \in U$. Затем выберем в $\left\langle g_{n}\right\rangle_{n \in \mathbb{N}}$ подпоследовательность $\left\langle h_{n}\right\rangle_{n \in \mathbb{N}}$ такую, что $u_{2}\left(h_{n}\right)=u_{2}$ для некоторого $u_{2} \in U$, и т. д. Лемма доказана.

Именно из последовательности произведений $\left\langle u_{1} \ldots u_{n}\right\rangle_{n \in \mathbb{N}}$ мы будем выбирать элемент $b$. Для этого нужно лишь выбрать номер $n$, но это потом. А пока что выберем натуральное $k$ так, чтобы для любых $g \in H, l(g)>k, x, y \in X$ переход

$$
g \rightarrow x g x \rightarrow y x g x y \rightarrow g x y(y x)^{-1}=g[x, y]
$$

миновал $F$.

Элемент $a$ выберем следующей леммой.

Лемма 2. Существует әлемент $a \in H, l(a)>k$, такой, что $l\left(a u_{1} \ldots u_{n}\right)>k u$ $l\left(u_{1} \ldots u_{n} a^{-1}\right)>k$ для $\operatorname{\theta cex} n$.

Первое условие означает, что последовательное навешивание справа на элемент $a$ коммутаторов $u_{1}, u_{2}, \ldots$ из нашей последовательности оставляет длину элемента $>k$, а значит, переход от $a$ к $a b$ будет миновать $F$. Второе условие соответствует свертыванию.

ДокАЗАТЕЛЬСтво. Допустим противное. Тогда для любого $a \in H$ найдется $n \in \mathbb{N}$ такое, что либо $a u_{1} \ldots u_{n} \in \Gamma(k)$, либо $u_{1} \ldots u_{n} a^{-1} \in \Gamma(k)$, где $\Gamma(k)=\{g \in H: l(g) \leqslant k\}$. Значит, для любого $a \in H$ либо $a \in\left(u_{1} \ldots u_{n} \Gamma(k)\right)^{-1}$, либо $a \in \Gamma(k) u_{1} \ldots u_{n}$. А значит,

$$
H=\left\{\left(u_{1} \ldots u_{n} g\right)^{-1}, g u_{1} \ldots u_{n}: n \in \mathbb{N}, \quad g \in \Gamma(k)\right\}
$$

Из этого следует, что

$$
\Gamma(n) \subseteq\left\{\left(u_{1} \ldots u_{i} g\right)^{-1}, g u_{1} \ldots u_{i}: i \leqslant n+k, \quad g \in \Gamma(k)\right\}
$$

Положим $\gamma(n)=|\Gamma(n)|$. Очевидно, $\gamma-$ функция роста $H$ и

$$
\gamma(n) \leqslant 2 \gamma(k)(n+k)
$$

Таким образом, рост $H$ линейный. Но тогда $H$ почти циклическая, - противоречие. Лемма доказана.

Номер $n$ выберем так, чтобы переход от $a u_{1} \ldots u_{n}=a b$ к $b a^{-1}$ миновал $F$. В результате общий переход от $a$ к $a^{-1}$ также будет миновать $F$. Теорема доказана.

СледСтвиЕ. Свободная әруппа с двумя образующими и любая бесконечная конечно порожденная периодическая группа не являются асимметрично разложсимыми.

Отметим, что для свободной группы с двумя образующими доказать то, что она не является асимметрично разложимой, можно и попроще, не привлекая соображений, связанных с ростом группы. 


\section{СПИСОК ЦИТИРОВАННОЙ ЛИТЕРАТУРЫ}

1. Протасов И. В. // Матем. заметки. 1996. Т. 59. №3. С. 468-471. 2. Banakh Т., Protasov I. // Вопросы алгебры. 2000. 3. Gromov M. // Publ. Math. IHÉS. 1981. V. 53. P. 53-73. 4. Bass H. // Proc. London Math. Soc. 1972. V. 25. №4. P. 603-614. 